\title{
Improved Confinement in JET hybrid discharges
}

\author{
J. Hobirk ${ }^{1}$, F. Imbeaux ${ }^{2}$, F. Crisanti ${ }^{3}$, P. Buratti ${ }^{3}$, C. D. Challis ${ }^{4}$,
} E. Joffrin ${ }^{2,5}$, B. Alper ${ }^{4}$, Y. Andrew ${ }^{4}$, P. Beaumont ${ }^{4}$, M. Beurskens ${ }^{4}$, A. Boboc ${ }^{4}$, A. Botrugno ${ }^{3}$, M. Brix ${ }^{4}$, G. Calabro' ${ }^{3}$, I. Coffey ${ }^{6}$, S. Conroy $^{7}$, O. Ford ${ }^{4}$, D. Frigione ${ }^{3}$, J. Garcia ${ }^{2}$, C. Giroud ${ }^{4}$, N. C. Hawkes ${ }^{4}$, D. Howell ${ }^{4}$, I. Jenkins ${ }^{4}$, D. Keeling ${ }^{4}$, M. Kempenaars ${ }^{4}$, H. Leggate ${ }^{4}$, P. Lotte ${ }^{2}$, E. de la Luna ${ }^{5}$, G. P. Maddison ${ }^{4}$, P. Mantica ${ }^{8}$, C. Mazzotta $^{3}$, D. C. McDonald ${ }^{4}$, A. Meigs ${ }^{4}$, I. Nunes ${ }^{9}$, E. Rachlew ${ }^{10}$, F. Rimini ${ }^{5}$, M. Schneider ${ }^{2}$, A. C. C. Sips ${ }^{5}$, J. K. Stober ${ }^{1}$, W. Studholme ${ }^{4}$, T. Tala ${ }^{11}$, M. Tsalas ${ }^{12}$, I. Voitsekhovitch ${ }^{4}$, P. C. de Vries ${ }^{12}$, and JET EFDA contributors $\ddagger$

JET-EFDA, Culham Science Centre, OX14 3DB, Abingdon, UK

${ }^{1}$ Max-Planck-Institut für Plasmaphysik, EURATOM Association, Boltzmannstr. 2, 85748 Garching

${ }^{2}$ CEA, IRFM, F-13108 Saint-Paul-lez-Durance, France

${ }^{3}$ ASSOCIAZIONE EURATOM-ENEA sulla Fusione C.R. FRASCATI, CP65, 00044 Frascati - ITALY

${ }^{4}$ EURATOM/CCFE Fusion Association, Culham Science Centre, Abingdon,OX14 3DB, UK

5 EFDA CSU Culham, Culham Science Centre, Abingdon,OX14 3DB, UK

${ }^{6}$ Queen's University, Belfast, BT7 1NN, UK

7 Association Euratom, Dept. Physics and Astronomy, Uppsala University, SE 75121 Uppsala

${ }^{8}$ Instituto di Fisica del Plasma, Associazone EURATOM ENEA -CNR, Milano, Italy

${ }^{9}$ Instituto de Plasmas e Fuso Nuclear, Associao EURATOM-IST, 1049-001 Lisboa, Portugal

${ }^{10}$ Association Euratom-VR, Dept. Phys., KTH. SE 10691, Stockholm Sweden

11 Association Euratom-Tekes, VTT, P.O. Box 1000, 02044 VTT, Finland

${ }^{12}$ FOM institute Rijnhuizen, EURATOM association, P.O. Box 1207, 3430BE, Nieuwegein, Netherlands

E-mail: joerg.hobirk@ipp.mpg.de

PACS numbers: 52.25.Fi,52.25.Xz,52.55Fa

\begin{abstract}
A new technique has been developed to produce plasmas with improved confinement relative to the $H_{98, y 2}$ scaling law [1] on the JET Tokamak. In the mid size Tokamaks ASDEX Upgrade and DIII-D heating during the current formation is used to produce a flat $q$-profile with a minimum close to 1 . On JET this technique leads to $q$-profiles with similar minimum $q$ but opposite to the other Tokamaks not to an improved confinement state. By changing the method utilising a faster current ramp with temporary higher current than in the flattop (current over shoot) plasmas with improved confinement $\left(H_{98, y 2}=1.35\right)$ and good stability $\left(\beta_{N} \approx 3\right)$ have been produced and extended to many confinement times only limited by technical constraints. The increase in $H_{98, y 2}$-factor is stronger with more heating
\end{abstract}

\$ See the Appendix of F. Romanelli et al., Proceedings of the 23rd IAEA Fusion Energy Conference 2010, Daejeon, Korea 
power as can be seen in a power scan. The $q$-profile development during the high power phase in JET is reproduced by current diffusion calculated by TRANSP and CRONOS. Therefore the modifications produced by the current over shoot disappear quickly from the edge but the confinement improvement lasts longer, in some cases up to the end of the heating phase.

Over the past 13 years a new scenario called hybrid scenario or improved H-mode has emerged on the mid-size tokamaks ASDEX Upgrade [2, 3, 4] and DIII-D [5, 6]. This scenario combines a higher $q_{95}$ operation with improved confinement compared to the $H_{98, y 2}$ scaling law. Could this kind of scenario be reproduced on ITER, new possibilities would arise, e.g. high $Q$ operation at reduced $I_{P}[7,8]$. Hybrid scenario plasmas can have significantly improved confinement above the standard $H_{98, y_{2}} \mathrm{H}$-mode scaling but the physics basis remains somewhat unclear due to many possible explanations e.g. increased $\vec{E} \times \vec{B}$ produced by strong gradients in a high toroidal rotation, an increase in pedestal pressure [9] or a change in $s / q$ which can stabilise turbulence [10,11] or a combination of shear and rotation effects [12]. At JET [13] discharges similar to the ones from DIII-D and ASDEX Upgrade have been carried out in a high triangularity configuration $\left(\delta_{l}=0.41, \delta_{u}=0.43, \delta_{a v}=0.42\right.$ values averaged over heating phase) before the 2008/9 campaigns. The $q$-profile modification has been done with low power (0.5-1.2MW) lower hybrid current drive (LHCD). Careful analysis later has shown that the initial $q$-profile modification was lost quite rapidly and well before the high power/high beta phase has been established. The discharges were done at low current and high (normalised) density. Nevertheless also in these old pulses some of the characteristics e.g. the stability against neoclassical tearing modes (NTM) at high $\beta_{N}$ have been reproduced but the confinement was not improved significantly over the $H_{98, y 2}$ scaling.

New experiments in JET in a low triangularity configuration $\left(\delta_{l}=0.31, \delta_{u}=0.14, \delta_{a v}=\right.$ 0.23) with low plasma densities have been done to explore the differences between the JET discharges $\left(H_{98, y 2}=1\right)$ to discharges from other experiments $1<H_{98, y 2}<1.8$. The configuration has been chosen such that the results can be compared to earlier JET pulses [14] and to pulses from ASDEX Upgrade [15]. Furthermore the low plasma density expected from H-mode discharges in this low triangularity configuration results in higher temperatures and longer current diffusion time scales so that possible transient effects can be better observed. The methods developed for the low triangularity configuration has later been ported to plasmas in a high triangularity configuration $\left(\delta_{l}=0.33, \delta_{u}=0.38, \delta_{a v}=0.36\right)$ and higher density which has been reported in [16]. The experiment started from the assumption that the central part of the $q$-profile plays an important role in the explanation of the observed confinement differences between the different tokamaks. In ASDEX Upgrade this has been shown for some cases of different confinement in similar discharges [10].

In this paper experimental results from the JET tokamak are presented. In the first paragraph the used $q$-profile modification method will be introduced. In the second paragraph the experimental results of the changed plasmas in terms of confinement are described. In the third one the discussion is extended to profile effects and in the fourth paragraph the properties with changing heating power are presented. In the fifth paragraph the $q$-profile evolution is compared to calculations from TRANSP and CRONOS and in the last paragraph 
a summary and discussion is presented. The paper concentrates on the experimentalfindings, a full theoretical treatment is beyond the scope of this paper.

\section{1. $q$-profile modification}

In the new experiments different experimental strategies to modify the central part of the $q$ profile have been tried. The first method utilised early NBI heating similarly as it is done on ASDEX Upgrade and DIII-D. Two different waveforms were tested, firstly a long NBI prelude with low power NBI (3-5MW) starting at $t=2 s$ and secondly a short NBI prelude starting at $t=3-3.5 s$ but with $P_{\mathrm{NBI}}=10 \mathrm{MW}$. The $P_{\mathrm{NBI}}$ is increased to values of $16-22 \mathrm{MW}$ at the time of the current flattop at $t=4 \mathrm{~s}$. In both cases it was possible to delay the onset of sawteeth significantly and to flatten the central part of the $q$-profile. The normalised confinement did not change significantly. The next idea tried was to use ion cyclotron heating in H-minority regime (ICRH) at approximately half radius from $t=2 s$ on. The main idea was to broaden the electron temperature profile and consequently the ohmic current profile which in the absence of external current drive is dominating the total current profile. Unfortunately the electron temperature profile shape did not change by much and only the higher temperature has let to a higher $\mathrm{q}$ at the start of the main heating but again not to changed confinement properties. It could not been clarified up to now whether some modification of the assumed heating deposition e.g. by mode conversion took place or if the stiffness was strong enough to produce peaked profiles with the very low power flux due to the remaining central ohmic heating. The last method tested in this series was to change the current ramp rate from $200 \mathrm{kA} / \mathrm{s}$ to $400 \mathrm{kA} / \mathrm{s}$ without applying additional heating. The introduced current density is located at the edge and needs to diffuse inwards for several seconds before it reaches the centre. Therefore if the current is risen fast then transiently more current is outside the plasma core and the current density profile becomes broader. In those pulses the current ramp was short enough to achieve a $q>1$ at the beginning of the main heating therefore no attempts to heat during the ramp were made. In some of those pulses an increased confinement up to $H_{98, y_{2}}=1.1$ has been observed but is still not as good as on other experiments. In addition most of the pulses had a large $n=2$ NTM if they were able to reach $\beta_{N}>2$. In some cases a $n=1$ NTM at higher beta was observed as well.

Analysis with the transport code TRANSP [17] have indicated that all these methods do not produce a $q$-profile which is as broad as on ASDEX Upgrade measured by the radius of the $q=1.5$ surface as seen as $3 / 2$ NTM position [10]. The current density profile produced by the faster current ramp is already relatively flat and it seemed not to be possible to extend the low shear region by just redistributing central current densities. As a result of this consideration a different method was tried. The aim was to produce in the core a $q$-profile which is very flat as in a low $q_{95}$ plasma by ramping to a higher current than intended. To achieve the right edge $q$ the current is ramped down after a short delay and the shear at the edge is increased. Due to the longer time scales for the core, the central current density does not change much during the current ramp down. As a last step the heating is switched on and the higher temperature in the core will lead to an even slower current diffusion and the state can be kept for a few 


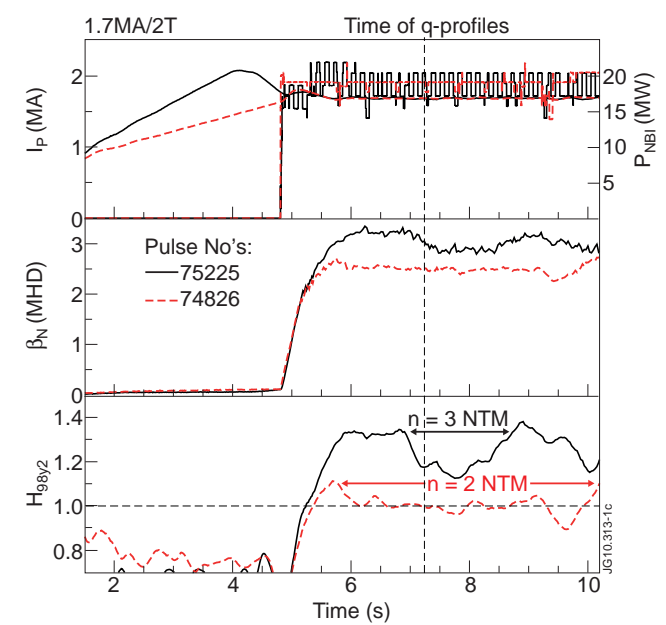

Figure 1. Time traces of a JET hybrid discharge with current ramp down before the main heating (current overshoot) in black compared to a pulse without strong $q$-profile modification in a dashed red line. In the upper graph the traces of the plasma current and the NBI heating power are shown. In the middle the normalised beta $\beta_{\mathrm{N}}$ and in the lower graph the $H_{98, y 2}$-factor is drawn.

seconds. The designed current "overshoot" to change the outer and central part of the $q$-profile can be seen in figure 1 with the plasma current trace in black. The $q$-profile at the time of the heating can be influenced by the initial current ramp rate, the length of the first plateau of the current over shoot and the ramp down rate. The achieved $q_{0}$ for the experiment is strongly connected to the start time of the heating.

The chosen method works for the low plasma current JET pulses with a target $q_{95}=4$ well. The method itself could be ported even to ITER if $q_{95}=4$ is desired. Some drawbacks should be considered though. The combination of relatively low $q_{95}$ and the transition to high $l_{i}$ in the current ramp down without NBI heating can lead to mode locks or loss of shape control and consequently to a disruption. These kind of problems will be more challenging if the flattop $q_{95}$ is lower and the current ramp down proves to be essential.

\section{Improved confinement reached}

Improved confinement up to $H_{98, y 2}=1.4$ has been reached transiently by using the current "overshoot" method. By small modifications to the scenario the MHD stability and the improved performance could be extended to about $5 \mathrm{~s}$ duration. The duration was only limited by a temperature limit in the NBI duct. In figure 1 some of the characteristic data of such a pulse (\#75225) in comparison to a pulse without strong q-profile modification (\#74826) are shown. The dimensional parameters of discharges discussed can be found in table 1 and the dimensionless parameters in table 2. For those two pulses the line averaged densities are similar, the same plasma shape is used and the NBI heating starts at the same time. Consequences of the change in $q$-profile are higher n-number NTMs in the plasma but also the global confinement as indicated by the $H_{98, y 2}$ factor is different. In figure 2 the $q$-profile from EFIT constrained by MSE and pressure data (including fast ion pressure) at $2.45 \mathrm{~s}$ after 


\begin{tabular}{|c|c|c|c|c|c|c|c|c|c|}
\hline Pulse & Time & $I_{P}$ & $B_{T}$ & $\bar{n}_{e}$ & $P_{\mathrm{NBI}}$ & $\beta_{N}^{\text {tot }}$ & $W_{\text {core }}$ & $W_{\text {pedestal }}$ & $\omega_{\text {tor }}$ \\
\hline & {$[\mathrm{s}]$} & {$[\mathrm{MA}]$} & {$[\mathrm{T}]$} & {$\left[10^{19} \mathrm{~m}^{-3}\right]$} & {$[\mathrm{MW}]$} & & {$[\mathrm{MJ}]$} & {$[\mathrm{MJ}]$} & $\mathrm{krad} / \mathrm{s}$ \\
\hline 75225 & 7.25 & 1.7 & 2 & 3.24 & 18.8 & 2.92 & $1.83 \pm 0.90$ & $1.02 \pm 0.24$ & 77.2 \\
74826 & 7.25 & 1.7 & 2 & 3.12 & 19.2 & 2.52 & $1.68 \pm 0.81$ & $0.80 \pm 0.24$ & 65.3 \\
75225 & $6 \pm 0.2$ & 1.7 & 2 & 3.6 & 18.8 & 3.07 & $1.98 \pm 0.30$ & $1.02 \pm 0.09$ & 76.7 \\
74826 & $5.6 \pm 0.1$ & 1.7 & 2 & 3.35 & 19.2 & 2.58 & $1.65 \pm 0.43$ & $0.83 \pm 0.14$ & 55.5 \\
79630 & $7 \pm 0.2$ & 1.7 & 2 & 3.56 & 17.5 & 2.55 & $1.64 \pm 0.41$ & $0.98 \pm 0.27$ & 77.8 \\
79628 & $7 \pm 0.2$ & 1.7 & 2 & 3.2 & 17.5 & 2.83 & $1.85 \pm 0.39$ & $1.07 \pm 0.25$ & 82.4 \\
73306 & $8.1 \pm 0.2$ & 2 & 2.4 & 3.51 & 20 & 2.21 & $2.24 \pm 0.48$ & $0.93 \pm 0.24$ & 79.8 \\
74836 & $8.1 \pm 0.2$ & 2 & 2.4 & 3.9 & 22 & 2.63 & $2.32 \pm 0.43$ & $1.29 \pm 0.14$ & 75.7 \\
75625 & $7.5 \pm 0.2$ & 1.7 & 2 & 3.1 & 17 & 2.8 & $2.18 \pm 1.14$ & $0.79 \pm 0.57$ & 86.2 \\
75626 & $7.5 \pm 0.2$ & 1.7 & 2 & 3.1 & 9.4 & 1.8 & $1.32 \pm 0.81$ & $0.69 \pm 0.41$ & 53.8 \\
75627 & $7.5 \pm 0.2$ & 1.7 & 2 & 3.0 & 13.5 & 2.26 & $1.89 \pm 0.87$ & $0.44 \pm 0.43$ & 66.4 \\
\hline
\end{tabular}

Table 1. List of dimensional 0d parameters for all pulses and used times and average interval. Toroidal angular frequency measured at $R=3.4 \mathrm{~m}$.

\begin{tabular}{|c|c|c|c|c|c|c|c|c|c|c|}
\hline Pulse & Time & $H_{98, y 2}$ & $\rho^{*}$ & $v^{*}$ & $\beta_{N}^{\text {th }}$ & $q_{95}$ & $R / L_{T_{i}}$ & $M$ & MHD & CuOv \\
\hline & {$[\mathrm{s}]$} & & $10^{-3}$ & $10^{-3}$ & & & & & & \\
\hline 75225 & 7.25 & 1.20 & 5.87 & 12 & 1.96 & 3.94 & 6.2 & 0.47 & $\mathrm{n}=3, \mathrm{FB}$ & Yes \\
74826 & 7.25 & 1.01 & 5.32 & 19 & 1.52 & 3.77 & 6.3 & 0.42 & $\mathrm{n}=2, \mathrm{n}=3$ & No \\
75225 & $6 \pm 0.2$ & 1.35 & 5.69 & 12 & 2.16 & 3.96 & 6.6 & 0.49 & - & Yes \\
74826 & $5.6 \pm 0.1$ & 1.10 & 5.34 & 20 & 1.65 & 3.77 & 7.3 & 0.4 & $\mathrm{n}=1, \mathrm{n}=3$ & No \\
79630 & $7 \pm 0.2$ & 1.23 & 5.48 & 18 & 1.88 & 3.87 & 6.4 & 0.52 & $\mathrm{FB}, \mathrm{n}=4$ & No \\
79628 & $7 \pm 0.2$ & 1.35 & 5.89 & 12 & 2 & 3.94 & 6 & 0.48 & $\mathrm{FB}, \mathrm{n}=5$ & Yes \\
73306 & $8.1 \pm 0.2$ & 1.07 & 5.09 & 13 & 1.53 & 3.8 & 6.5 & 0.47 & $\mathrm{n}=3, \mathrm{n}=5$ & No \\
74836 & $8.1 \pm 0.2$ & 1.20 & 5.13 & 13 & 1.81 & 3.95 & 6.5 & 0.43 & $\mathrm{FB}, \mathrm{n}=4, \mathrm{n}=5$ & Yes \\
75625 & $7.5 \pm 0.2$ & 1.31 & 5.9 & 12 & 1.9 & 3.95 & 6.7 & 0.5 & $\mathrm{FB}, \mathrm{n}=4, \mathrm{n}=5$ & Yes \\
75626 & $7.5 \pm 0.2$ & 1.10 & 4.93 & 23 & 1.32 & 3.77 & 5.6 & 0.45 & $\mathrm{n}=1$ & Yes \\
75627 & $7.5 \pm 0.2$ & 1.17 & 5.45 & 15 & 1.56 & 3.84 & 6.3 & 0.48 & $\mathrm{FB}, \mathrm{n}=1, \mathrm{n}=4$ & Yes \\
\hline
\end{tabular}

Table 2. List of non-dimensional 0d parameters for all pulses and used times and average interval. $R / L_{T_{i}}$ and the Mach number (defined as $M=\sqrt{\frac{m}{e}} \frac{v}{\sqrt{T_{\mathrm{i}}}}$ ) are measured at $R=3.4 \mathrm{~m}$. MHD information includes only core MHD and $\mathrm{n}=1$ is also $\mathrm{m}=1, F B$ is the abbreviation for fishbones and Saw is the abbreviation for sawteeth. The label $\mathrm{CuOv}$ indicates whether the current overshoot technique was used in this pulse or not.

start of the heating is shown. In addition the positions of MHD modes from different times are also shown. For this the mode number is determined by the magnetic pick up coils and a corrected mode frequency is mapped using the measured toroidal rotation to a position. The $q$-profile of $\# 75225$ has a large low shear region up to $R=3.4 \mathrm{~m}$ compared to \#74826. Also the positions of the critical rational $q$ surfaces for NTM stability (as indicated in figure 2) are moved significantly outward in \#75225. The density in \#75225 is higher at $t=6 \mathrm{~s}$ but relaxing later in the pulse to the same values as in \#74826 at $t=5.6 \mathrm{~s}$. Many parameters in \#74826 


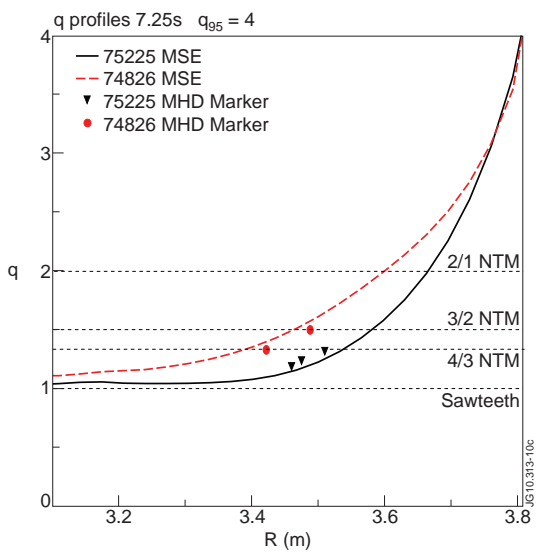

Figure 2. Equilibrium reconstructed $q$ profile $2.45 \mathrm{~s}$ after start of the main heating for a pulse with current overshoot in black and a pulse without in dashed red.

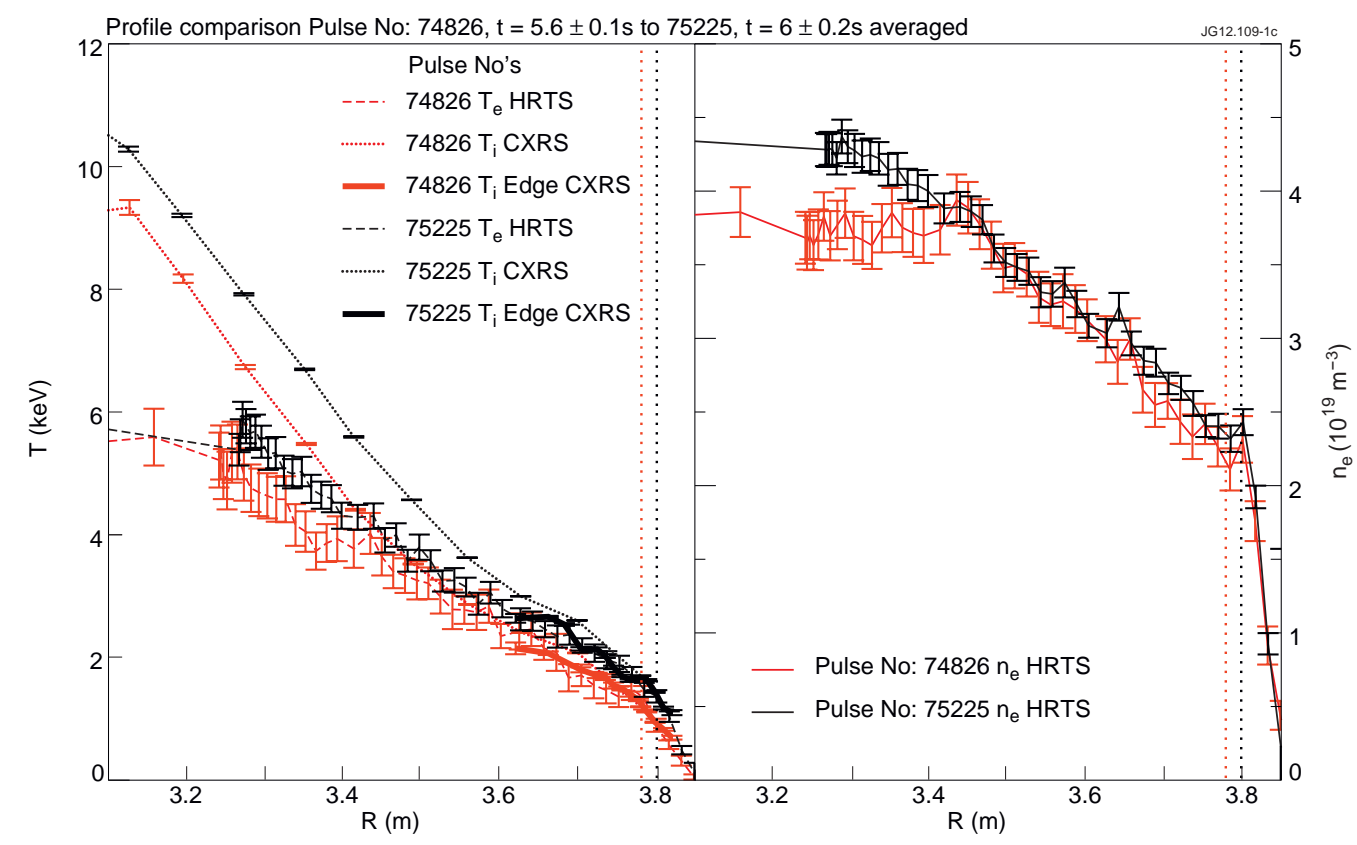

Figure 3. Temperature profiles from a pulse with modified $q$-profile in black (current overshoot) and without modified $q$-profile in red. The profile in closed lines are ion temperatures measured by Charge Exchange Recombination Spectroscopy (CXRS). Closed thick lines are from the edge CXRS system. Dashed lines are electron temperatures measured by the High Resolution Thomson Scattering (HRTS) diagnostic. On the right hand side the corresponding density profiles from HRTS. The pedestal radius used to derive the pedestal energy for table 1 is indicated as dotted line in the appropriate colour.

are dominated by the existence of the $n=2$ NTM (a very weak $n=3$ from $5.4 \mathrm{~s}$ to the end of the heating exists as well), in particular stored energy and plasma density. Therefore it is difficult to compare the time traces to the ones of $\# 75225$ wherea $n=3$ NTM is active (very weak $n=4$ and $n=5$ modes exist from $5 \mathrm{~s}$ on and disappear with the occurrence of the $n=3$ NTM) and only for a comparatively short time. As a consequence all the following profile comparison is done 
for an early time point which is NTM free in both discharges but might still be transient. If the comparison is done at $\mathrm{t}=5.6 \mathrm{~s}$ in the discharge the effects are still visible but the differences are smaller.

\section{Differences in kinetic profiles}

In the following it will be discussed how the improved confinement is reflected in terms of profile development. The most common case is an improvement in pedestal and core confinement as discussed in the first subsection. An only pedestal pressure improvement is discussed in the second subsection and an only core profile increase in the last subsection. In the following $W_{\text {pedestal }}$ is determined by integrating the pressure profile outside the $\Psi_{\text {pedestal }}$ and taking the value of the pressure at $\Psi_{\text {pedestal }}$ constant inside. $\Psi_{\text {pedestal }}$ is defined here as the poloidal flux where the second derivative of the pressure becomes smallest within $\Psi \geq 0.8$. The result has been inspected visually and represents the pedestal top reasonably well. The error bars have been derived from the error bars of the pressure profile build up from the kinetic profiles. The error bars on the kinetic data are specific to the diagnostic used, mostly they are calculated e.g. from the differences between a fitted spectral line and the measured data. The profiles are averaged over some time interval. Assuming that the errors in the profile are statistical distributed, the error bars have been reduced accordingly. The resulting error in the pedestal stored energy is about $20 \%$ using the HRTS diagnostic. The error bars do not include uncertainties in the equilibrium reconstruction nor in the positioning of the pedestal or systematic errors. Qualitatively an assumption of a fixed position in flux e.g. $\Psi_{\text {pedestal }}=0.9$ results in a similar picture. Only in the later discussed cases (\#75625-7) with pressure profiles build with the help of LIDAR data the $\Psi_{\text {pedstal }}$ becomes uncertain because the spatial resolution is not sufficient and the error bars increase to $60-100 \%$ in the pedestal energy.

\subsection{Pedestal and core improvement}

The electron and ion temperature (see figure 3) in \#75225 are higher for any radius and the electron density is higher as well for any radius compared to \#74826. The differences in the kinetic pressure seen in figure 3 explain within $5 \%$ the difference in H-factor in table 2. The differences clearly start in the H-mode pedestal and are then propagated towards the centre as reported for ASDEX Upgrade improved H-mode [9]. The inverse gradient length and the Mach number are listed in table 2. The inverse gradient length is slightly higher for the pulse with lower normalised confinement but the Mach number is slightly lower. The ion temperature profile is not constant during the pulse. Even the ion temperature gradient length varies with time and radius therefore it is difficult to make a straight conclusion on changes of transport.

\subsection{Pedestal improvement}

Unfortunately the profile changes shown by figure 3 are not always the case. In figure 4 profiles are shown for a pair of pulses done at 2MA/2.4T. Pulse \#73306 has been done with 


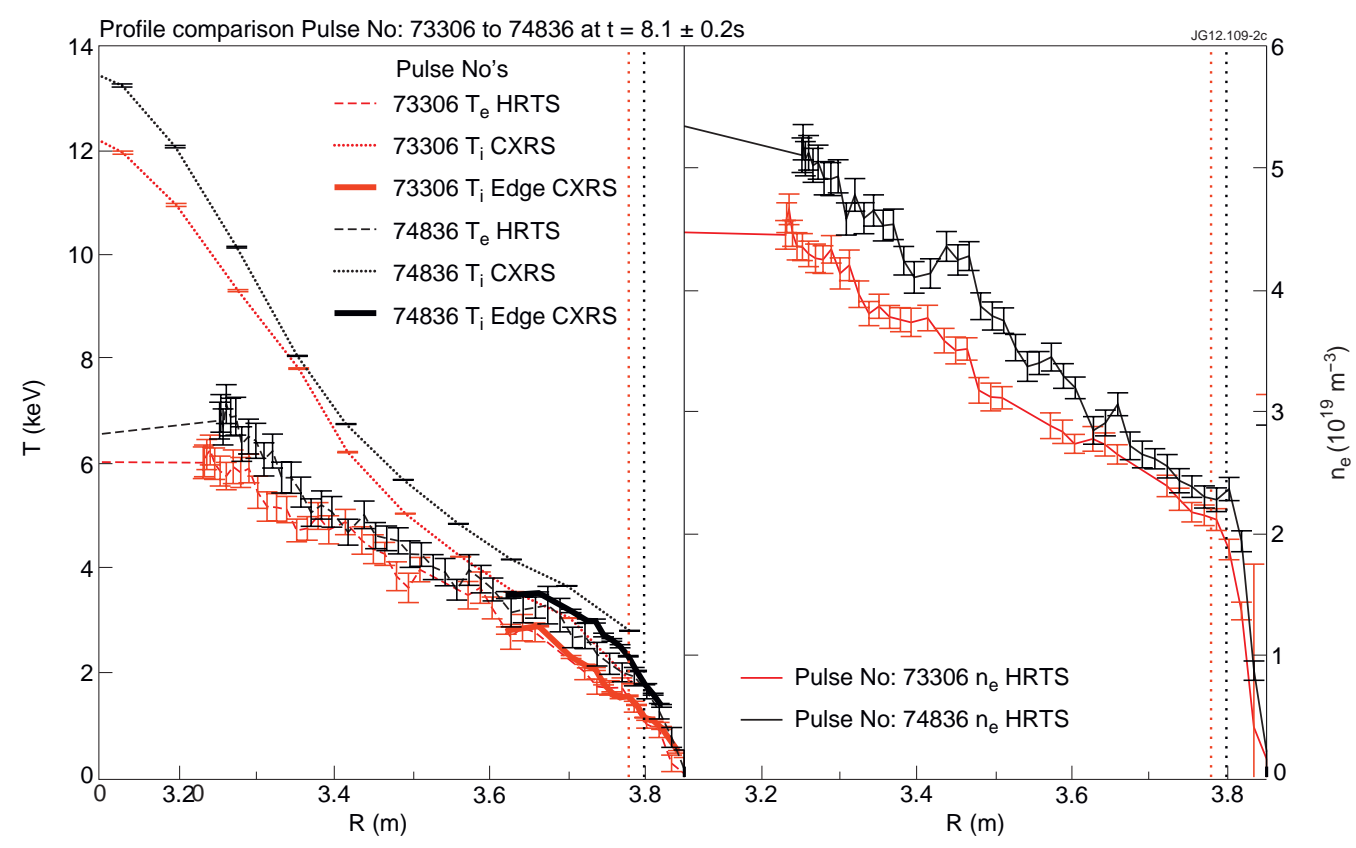

Figure 4. Temperature profiles from a pulse with modified $q$-profile in black (current overshoot) and without modified $q$-profile in red. The profile in closed lines are ion temperatures measured by Charge Exchange Recombination Spectroscopy (CXRS). Closed thick lines are from the edge CXRS system. Dashed lines are electron temperatures measured by the High Resolution Thomson Scattering (HRTS) diagnostic. On the right hand side the corresponding density profiles from HRTS. The pedestal radius used to derive the pedestal energy for table 1 is indicated as dotted line in the appropriate colour.

some NBI preheat but without current overshoot $\left(H_{98, y 2} \approx 1\right)$, the other pulse \#74836 has been done following the recipe developed at $1.7 \mathrm{MA} / 2 \mathrm{~T}$ with a current overshoot $\left(H_{98, y 2}=1.2\right)$. The profiles are now such that the stored energy in the core is constant and the whole difference can be attributed to a change in edge stored energy (see table 1).

\subsection{Core improvement}

In figure 5 profiles from another comparison pair at $1.7 \mathrm{MA} / 2 \mathrm{~T}$ are shown. In this case pulse \#79630 has been done without current overshoot and significantly delayed NBI injection to produce a $q$-profile as close as possible to fully diffused H-mode $q$-profile. The confinement time in the pulse without current over shoot is still larger than the scaling predicts $\left(H_{98, y 2}=1.2\right)$. The reason for this is not clear. The comparison pulse \#79628 has been done at the same plasma parameters as the earlier discussed pulse \#75225 $\left(H_{98, y 2}=1.35\right.$, see also table 1$)$ and performs even better. In this case the edge stored energy is the same for both pulses and the additional energy can be found in the plasma core. A possible mechanism is discussed in [12] based on the idea that the high rotation speed together with low magnetic shear in the core can influence the turbulence properties and can reduce the ion temperature stiffness. 


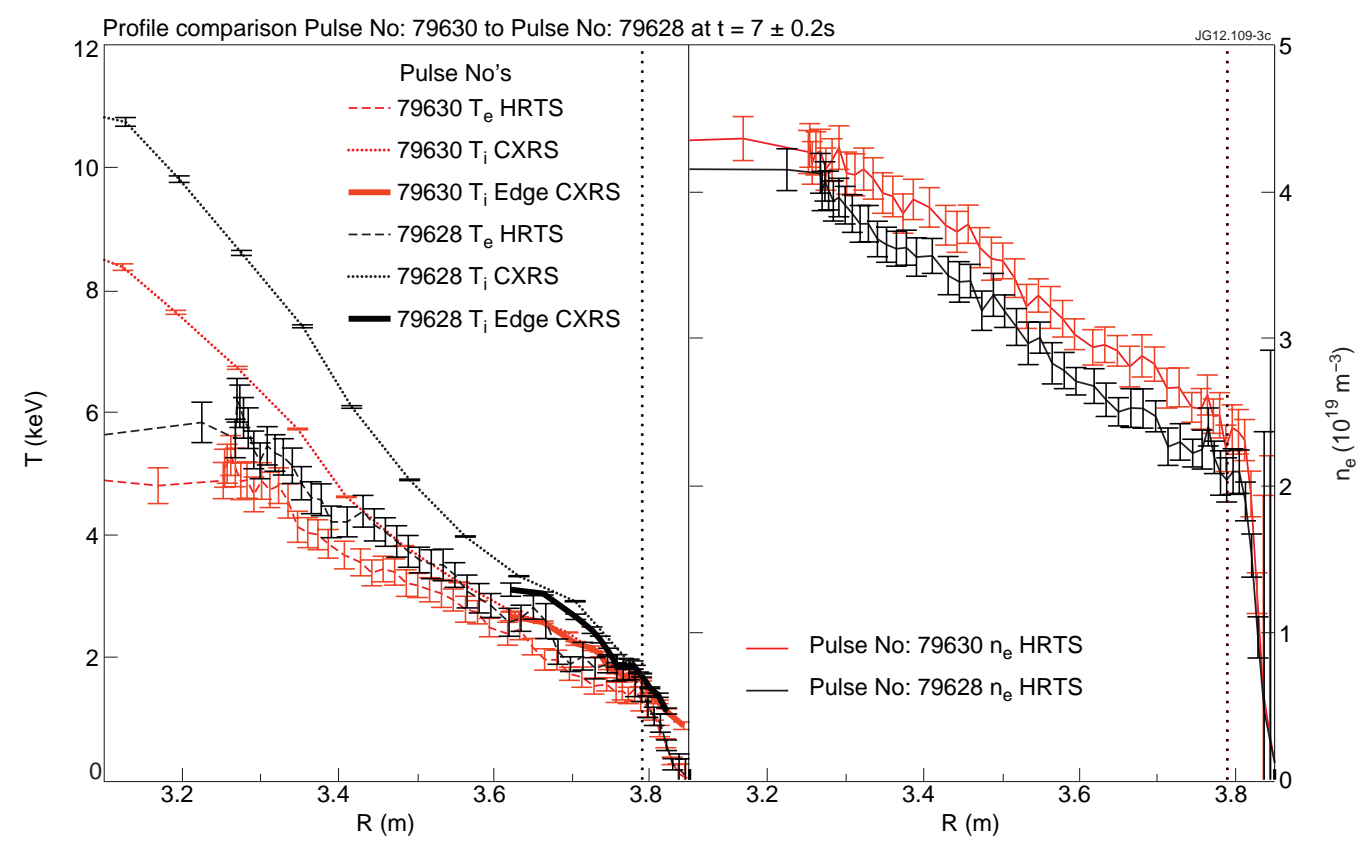

Figure 5. Temperature profiles from a pulse with modified $q$-profile in black (current overshoot) and without modified $q$-profile in red. The profile in closed lines are ion temperatures measured by Charge Exchange Recombination Spectroscopy (CXRS). Closed thick lines are from the edge CXRS system. Dashed lines are electron temperatures measured by the High Resolution Thomson Scattering (HRTS) diagnostic. On the right hand side the corresponding density profiles from HRTS. The pedestal radius used to derive the pedestal energy for table 1 is indicated as dotted line in the appropriate colour.

\subsection{Summary of profile effects}

The three cases presented show the difficulty to determine from which part of the profile the improved confinement comes. The total energy is most often changed by about $20-30 \%$ and several profiles can be different. Therefore it is difficult to pin down the changes in stored energy to certain profile effects. Nevertheless, in the cases with highest confinement the pedestal and the core profiles seem to be improved. But in other cases a pedestal improvement or a core improvement can be found. In the data in tables 2 and 1 there is a correlation between $q_{95}$ and the confinement improvement a the edge. As there is a correlation of the toroidal velocity and the core confinement. Now one needs to determine if the observed correlations are a cause or a consequence of the higher confinement.

a) The higher pedestal pressure leads to a higher bootstrap current close to the edge. Together with a constant total current this leads to an increase in current density at the edge and a decrease of the current density in the core, both will result in a lower $l_{i}$ value. As discussed before the shape is relatively sensitive to changes in $l_{i}$ and $\beta$. The change in the fatness and volume of the shape now results in a change in $q_{95}$ even though the plasma current and toroidal magnetic field are constant. Therefore we explain the change in $q_{95}$ as a consequence of the increased pedestal pressure.

b) More difficult to answer is the cause or consequence question for the rotation. On the one 
hand, there are physics processes known e.g. $\vec{E} \times \vec{B}$ shearing which reduce the turbulence strength and would be a prime candidate to explain the confinement enhancement in the core. Another, more complicated mechanism is discussed by reference [14]. Where a high rotational shear together with small magnetic shear can lead to reduction of stiffness. On the other hand, it is often (almost always) observed that an increase in ion temperature (gradient) is accompanied by an increase in rotation speed. A physics explanation for this could be that the momentum transport is produced by the same micro turbulence as the ion heat transport. In this case it would be clear that with the same momentum and heat source the ion temperature and the toroidal rotation speed are increased in the same way if the confinement improvement is due to a reduction in micro turbulence (e.g. ITG and/or TEM) in the core. The confinement improvement cause could be unrelated to this e.g. being caused by a reduction in $s / q$ or $T_{i} / T_{e}$ ratio. The data base collected within this experiment is insufficient to answer this question. This could be helped e.g. by gyro fluid or better gyro kinetic calculations which are beyond the scope of this paper.

In the end up to now no criterion has been found to determine when the different improvements take place because the different discharges seem to be rather similar and the changes are often too small to draw a strong conclusion.

\section{Power scaling}

Another observation is that in this kind of pulses the confinement normalised to the $H_{98, y 2}$ scaling increases with increasing input power. This has been observed e.g. on ASDEX Upgrade [8], DIII-D [18] and JET [19]. On the other hand in dedicated experiments it was also found that the $H_{98, y 2}$ scaling law fits reasonably well [20]. The papers cited above use different data bases and include partially H-modes without active $q$-profile forming. In figure 6 the confinement properties of 3 different pulses are shown. The highest power pulse has been done at the same plasma parameters as the earlier example \#75225 (See also table 1). The other two pulses are a beta scan (the neutral beam injected power is feedback controlled on the measured diamagnetic poloidal beta) at similar plasma parameters otherwise, e.g. electron densities (feedback controlled but with very similar gas flow) and plasma shaping. The lowest power pulse exhibits a normalised confinement a little better than the scaling, the medium power case has a significant improved normalised confinement and the highest power has the best normalised confinement. Even though the experiment has been carried out as beta scan it is not easy to make a conclusion on the beta scaling because e.g. the normalised gyro radius $\rho^{*}$ is changing as well in this scan. Also the rotation speed does vary largely (similar to the ion temperature, see also table 1) due to the difference in NBI heating but the Mach number is similar. In figure 7 the kinetic profiles of the three different pulses for $t=7.5 \mathrm{~s}$ are shown. The line averaged electron density (See table 1) has been kept constant but a small tendency to peak centrally with increasing NBI power and fuelling is visible. It can also be seen that the gain in stored energy is mostly in the ion channel (as it has been seen on ASDEX Upgrade [21] as well). The increased heating power is mainly coupled to the ions for the beam energy used. The low densities lead to a weak coupling of the ions to the electrons, therefore this 


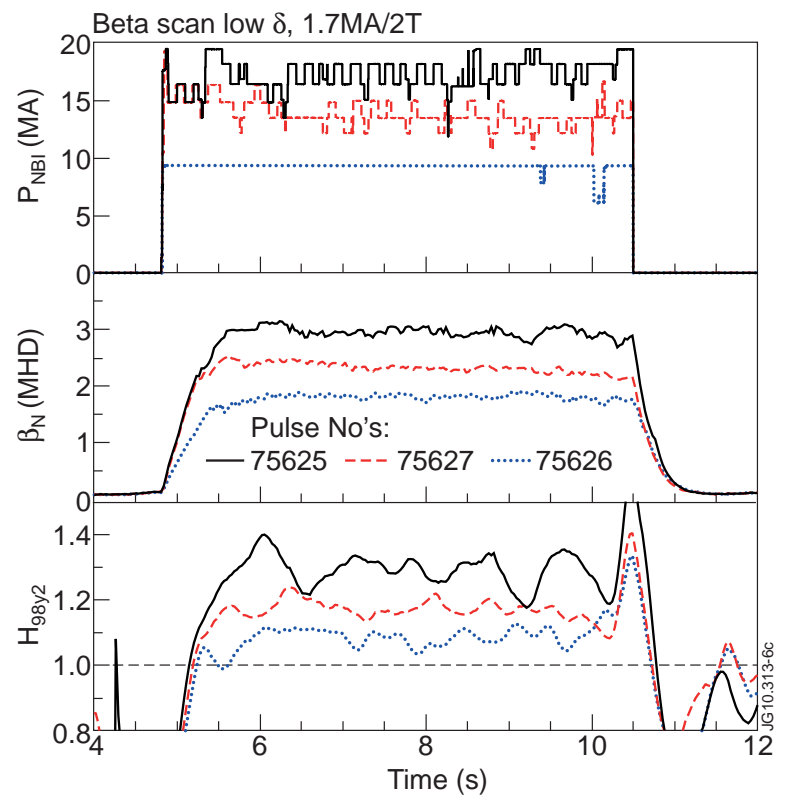

Figure 6. Time traces of three JET hybrid discharges with current overshoot in low triangularity. In the upper graph the traces of the NBI heating power are shown. In the middle the normalised beta $\beta_{\mathrm{N}}$ and in the lower graph the $H_{98, y_{2}}$-factor is drawn. The $H_{98, y 2}$ data is averaged over $400 \mathrm{~ms}$ and takes the time derivative of the stored diamagnetic energy into account, this leads to a not so constant numerical value during the pulse and an overestimation at the time when the NBI power is switched off.

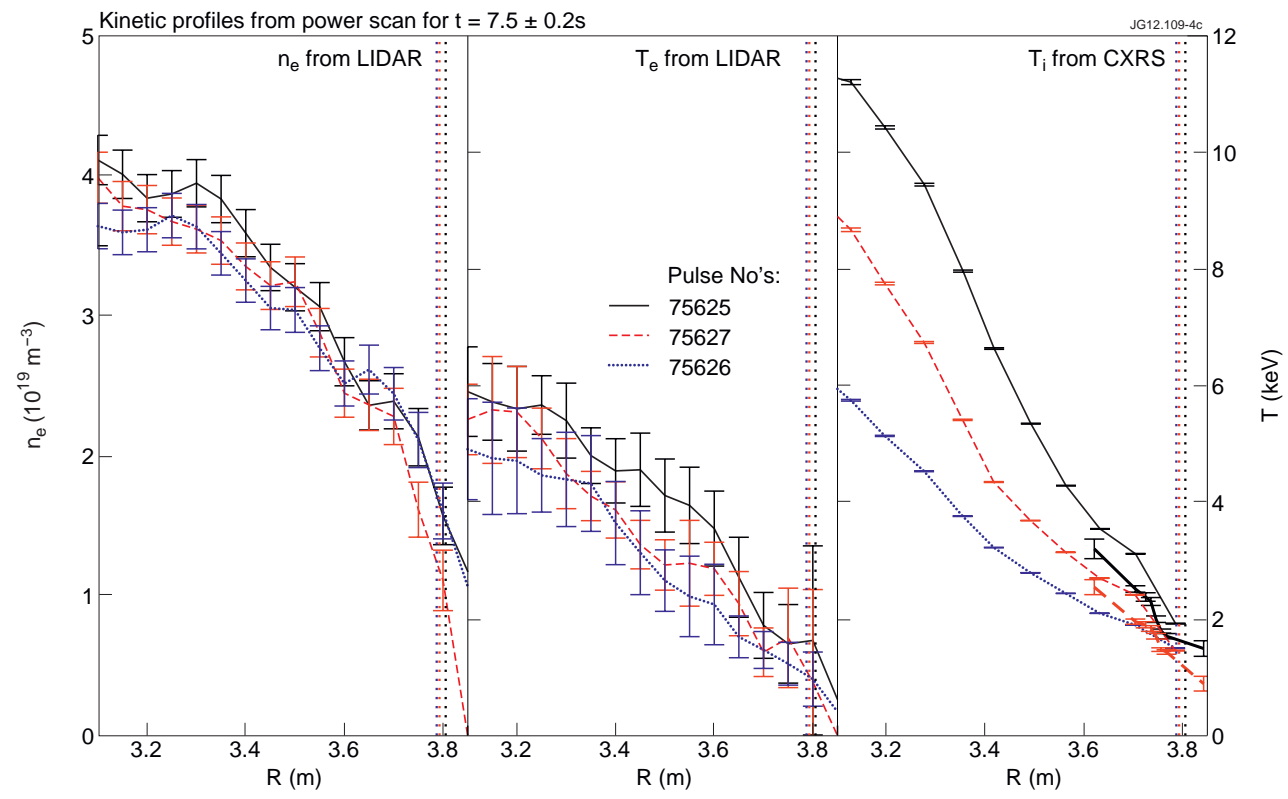

Figure 7. Kinetic profiles for the three JET hybrid discharges of figure 6 at $t=7.5 \mathrm{~s}$ using the same colour coding. On the left side the electron density, in the middle the electron temperature and on the right the ion temperature. The temperature profiles are plotted using the same scale. The pedestal radius used to derive the pedestal energy for table 1 is indicated as dotted line in the appropriate colour.

change of $T_{i} / T_{e}$ was almost expected. In the comparison of those pulses the heating power is not constant between pulses. In this case the pedestal (ion-) temperature is increasing 
Pulse No: 75225 q time traces at different radii and profile for $t=7.5 \mathrm{~s}$

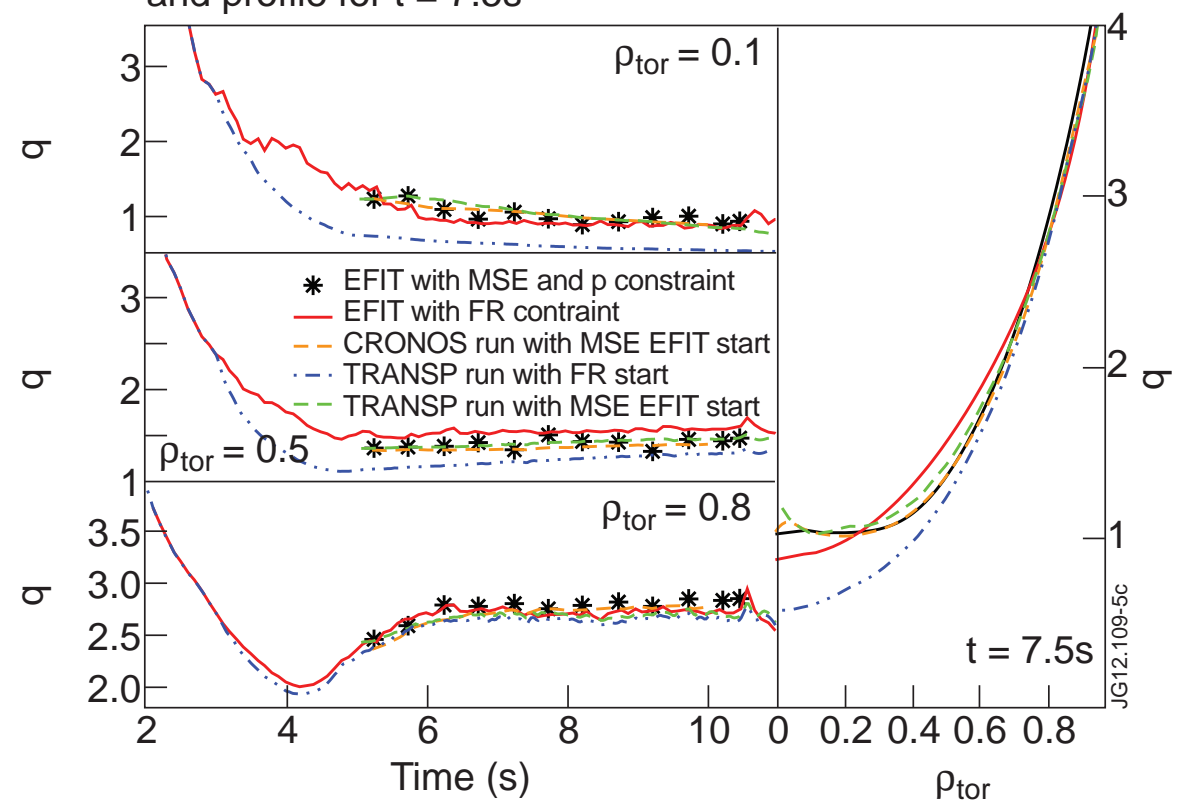

Figure 8. Time traces of $q$ at different $\rho_{\text {tor }}$ for a low triangularity discharge. The $q$-profile is modified using a current ramp down. MSE and pressure constrained equilibrium in black, a Faraday rotation in a red line, an interpretative TRANSP calculation starting from a Faraday rotation $q$-profile at $t=3 \mathrm{~s}$ in a dash dotted blue line, a interpretative TRANSP calculation starting from a MSE $q$-profile at $t=5.26 \mathrm{~s}$ in a green dashed line and a CRONOS run starting from a MSE $q$-profile at $t=5.26 \mathrm{~s}$ in a turquoise dashed line are plotted.

significantly. Using the shown kinetic profiles and calculating the pedestal contribution to the total kinetic stored energy the contribution is about $30 \%$ with large error bars. The calculated number for the low heating power discharge is slightly larger and amounts to $33 \%$ but the difference is not significant. The inverse ion temperature gradient length is increasing from the lowest to the highest power pulse.

\section{5. $q$-profile development}

On ASDEX Upgrade [8] and DIII-D [7] transport code calculations based on current diffusion using neo-classical resistivity are sometimes unable to predict the $q$-profile development. In the following five different $q$-profile time developments will be discussed. Firstly a EFIT calculation with MSE data and pressure profile constraints using a spline representation for the poloidal flux function (will be called in short MSE $q$ ). Secondly a EFIT calculation with Faraday rotation and interferometric density input without pressure constraint and using low order polynomials as representation for the poloidal flux function (in short FR $q$ ). There are some systematic differences between the FR $q$-profiles and the MSE $q$-profiles. Those differences reflect partially the uncertainties in the $q$-profile reconstruction in the sense that firstly the pressure constraint is missing, secondly the reconstructed electron density profile 
Pulse No: 74826 q time traces at different radii and profile for $t=7.5 \mathrm{~s}$

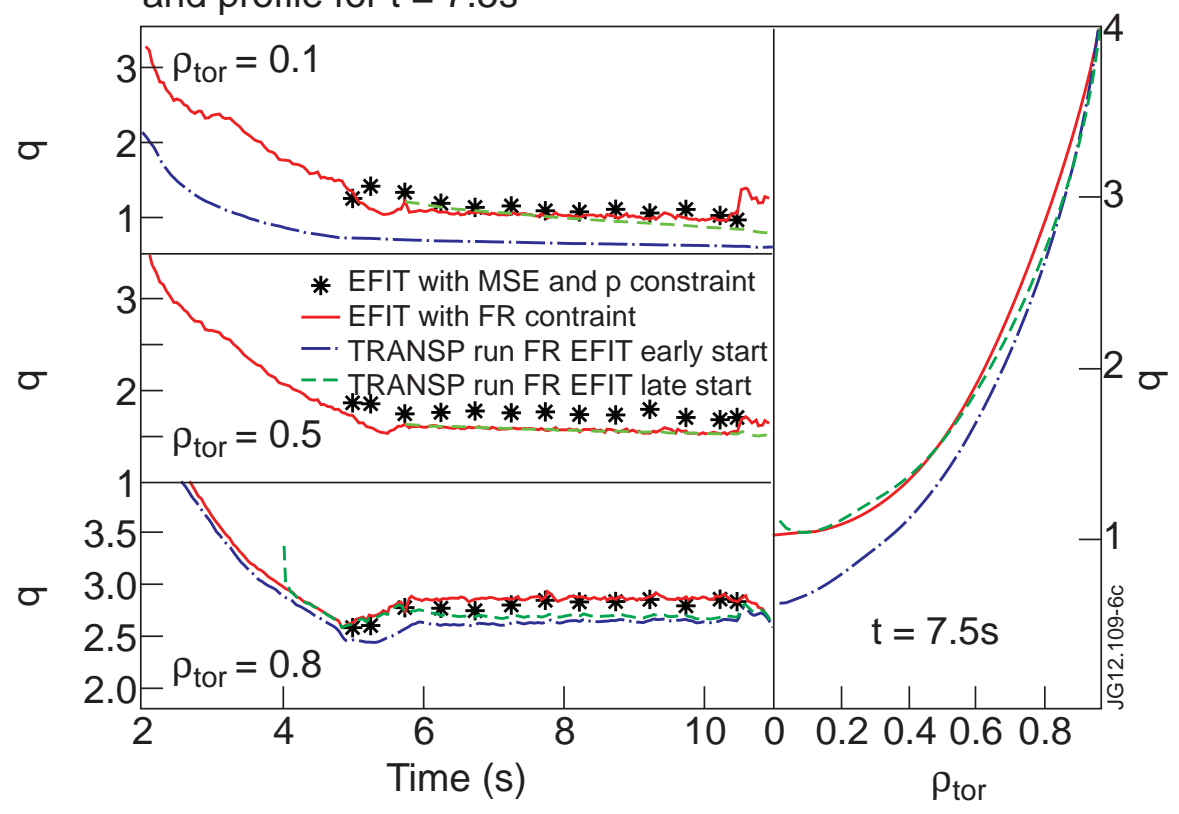

Figure 9. Time traces of $q$ at different $\rho_{\text {tor }}$ for a low triangularity discharge. The $q$ profile is not modified in the current ramp (H-mode reference). MSE and pressure constrained equilibrium in black, a Faraday rotation in a red line, an interpretative TRANSP calculation starting from a Faraday rotation $q$-profile at $t=3 \mathrm{~s}$ in a dash dotted blue line, a interpretative TRANSP calculation starting from a MSE $q$-profile at $t=5.26 \mathrm{~s}$ in a green dashed line and a CRONOS run starting from a MSE $q$-profile at $t=5.26 \mathrm{~s}$ in a turquoise dashed line are plotted.

within EFIT is not showing the full details because only 4 chords are used and thirdly due to the availability of only 5 chords of the Faraday rotation measurement a low order polynomial is used to represent the poloidal flux function. These effects make in this paper a FR $q$-profile less reliable than the MSE $q$-profile. On the other hand, due to the late heating the MSE diagnostic is not available before $t=5.26 \mathrm{~s}$ and the trend in the $q$ profile is quite consistent independent of the diagnostic except in the very core for a limited amount of time.

The third and fourth $q$-profiles were calculated by the TRANSP code using experimental data from HRTS $\left(n_{e}, T_{e}\right)$ and charge exchange diagnostic $\left(T_{i}, Z_{\text {eff }}\right)$ as input and using neoclassical resistivity, bootstrap current and a neutral beam current drive model as basis [17]. The difference between the two calculations is the starting $q$-profile which is a FR $q$ at $t=3 \mathrm{~s}$ in one case and a MSE $q$ at $t=5.26 \mathrm{~s}$ in the other case. The fifth $q$-profile was calculated by the transport code CRONOS [22] starting with the MSE $q$ and the same experimental profiles as used in TRANSP.

In the discussed plasma the confinement improvement appears to be initiated by a modification of the outer part of the $q$-profile by utilising a current overshoot technique. Naturally the fast change of $q$ by this transient method will disappear on a similar time scale as introduced with a delay caused by the increased temperature due to the applied strong heating. The time traces in figure 8 show that indeed most of the change in the outer part of the $q$-profile 
has been removed by current diffusion after about 2 s, however the confinement for \#75225 remains high. There is some small trend left (especially in the core) for the rest of the heating period - the $q$-profile does not reach steady state during the heating period. Qualitatively the TRANSP and CRONOS calculations starting with the first MSE $q$-profile reproduce the trend and indicate that classical current diffusion may be sufficient to explain the changes in $q$ within the measurement uncertainties during the heating phase. There are inconsistencies similarly to [24] during the ohmic phase which might be related to the reconstruction of the Faraday rotation data and/or the incomplete $Z_{\text {eff }}$ profile and/or physics outside the classical current diffusion calculation based on neoclassical resistivity.

A similar analysis for a pulse without current over shoot e.g. \#74826 is tainted by the occurrence of a low n-number NTM. Nevertheless in figure 9 a similar comparison as for the pulse with current overshoot is shown. If the TRANSP calculation is started in the heating phase the differences to the experimental data are not significant. In this pulse the first measurements of the MSE and the reconstructed $q$-profiles from it are not consistent with the existence of $\mathrm{m} / \mathrm{n}=1 / 1$ mode (no regular sawteeth) in the plasma. Therefore the late starting point for $q$ has been chosen from a FR constraint equilibrium. Also here inconsistencies in the ohmic phase especially in the core are observed.

\section{Summary and discussion}

Experiments were performed on JET to scan the $q$-profile with a central $q$ around 1 to see if a domain could be found where the confinement can be improved significantly compared to a plasma in H-mode without $q$-profile modification. Different methods have been tried to change the $q$-profile which mainly change the central part of the $q$-profile based on the assumption that a low shear in the centre allows the hybrid scenario to reach higher confinement. This approach was not successful on JET at first and a larger change in the core $q$-profile and a change in the outer part of the $q$-profile was necessary to improve the confinement significantly. On JET the chosen method up to now is a small current ramp down after a fast current ramp up and immediate strong heating following the current ramp down. The confinement improvement can affect the core and edge profiles without strong localisation in the core (e.g. not a strong localised transport barrier). An improved pedestal pressure together with reduced transport in the core leads to an enhanced confinement. The confinement enhancement survives the decay of the change in current profile in the outer part of the plasma by current diffusion by a significant amount of time. Calculations by TRANSP and CRONOS can follow the experimental $q$-profile evolution in the main heating phase showing that classical current diffusion is enough to explain the late $q$ evolution. This scenario operates at high normalised beta and therefore avoiding NTMs is a challenge. Nevertheless a relatively stable regime has been reached at low triangularity with only 5/4 or 4/3 NTMs but the extend of this operational window is not yet determined. Furthermore, is has been shown that in this scenario the normalised confinement improves with higher applied power. These plasmas show many similar properties to pulses from ASDEX Upgrade and DIII-D e.g. improved confinement with large $T_{i} / T_{e}$ at low density with high rotation and a flat $q$-profile in 
the centre. Also the tendency to have higher normalised confinement at higher heating power is similar. If the underlining physics mechanism are the same as in ASDEX Upgrade and DIIID hybrid plasma, then the scenario has been shown to be extendable to lower $\rho^{*}$, breaking the trend reported in [23] and hence showing potential to be ported to ITER. Remaining problems are mainly MHD related and may be less severe for ITER because of its ECCD capability allowing NTM stabilisation. However, many physics questions remain open including how the scenario can be used at higher densities, lower rotation speeds, with impurity seeding and whether continuous current drive would be necessary to maintain it.

\section{Acknowledgments}

This work was supported by EURATOM and carried out within the framework of the European Fusion Development Agreement. The views and opinions expressed herein do not necessarily reflect those of the European Commission.

[1] ITER Physics Expert Groups on Confinement and Transport and Confinement Modelling and Database,

ITER Physics Basics Editors, and ITER EDA, Nucl. Fusion 39, 2175 (1999).

[2] O. Gruber et al., Phys. Rev. Lett. 83, 1787 (1999).

[3] R. Wolf et al., Plasma Phys. Control. Fusion 41, B93 (1999).

[4] A. Staebler et al., Nucl. Fusion 45, 617 (2005).

[5] T. C. Luce et al., Nucl. Fusion 41, 1585 (2001).

[6] T. C. Luce et al., Physics of Plasmas 11, 2627 (2004).

[7] M. R. Wade et al., Nucl. Fusion 45, 407 (2005).

[8] A. Sips et al., Nucl. Fusion 47, 1485 (2007).

[9] C. F. Maggi et al., Nucl. Fusion 47, 535 (2007).

[10] J. Stober et al., Nucl. Fusion 47, 728 (2007).

[11] J. E. Kinsey, R. E. Waltz, and J. Candy, Physics of Plasmas 13, 022305 (2006).

[12] P. Mantica et al., Phys. Rev. Lett. 107, 135004 (2011).

[13] E. Joffrin et al., Proceedings of the 22nd IAEA Fusion Energy Conference, Geneva, Switzerland (2008).

[14] E. Joffrin et al., Nucl. Fusion 45, 626 (2005).

[15] A. Sips et al., Europhysics Conference Abstracts, Proc. 30th EPS Conference, St. Petersburg 2003 27A, O (2003).

[16] E. Joffrin et al., Proceedings of the 23nd IAEA Fusion Energy Conference, Daejon, Republic of Korea (2010).

[17] R. V. Budny et al., Physics of Plasmas 7, 5038 (2000).

[18] C. Petty et al., Physics of Plasmas 11, 2514 (2004).

[19] D. C. McDonald et al., Plasma Phys. Control. Fusion 46, A215 (2004).

[20] L. Vermare et al., Nucl. Fusion 47, 490 (2007).

[21] A. Manini et al., Nucl. Fus. 46, 1047 (2006).

[22] J. F. Artaud et al., Nucl. Fus. 50, 043001 (2010).

[23] D. C. McDonald et al., Plasma Phys. Control. Fusion 50, 124013 (2008).

[24] I. Jenkins et al., Europhysics Conference Abstracts, Proc. 37th EPS Conference, Dublin 2010, (2010). 